\title{
Immunohistochemical detection of steroid receptors in breast cancer: a working protocol
}

Robin Leake, Diana Barnes, Sarah Pinder, Ian Ellis, Liz Anderson, Tom Anderson, Ruth Adamson, Tony Rhodes, Keith Miller, Rosemary Walker on behalf of the UK Receptor Group, UK NEQAS, the Scottish Breast Cancer Pathology Group, and the Receptor and Biomarker Study Group of the EORTC

\begin{abstract}
Department of Biochemistry and Molecular Biology, Davidson Building, University of Glasgow, Glasgow G12 8QQ, UK $\mathrm{R}$ Leake
\end{abstract}

ICRF Breast Pathology Laboratory, Guy's Hospital, London SE1 9RT, UK

D Barnes

Department of Histopathology, City Hospital, Hucknall

Road, Nottingham NG5 1PB, UK

$S$ Pinder

I Ellis

Clinical Research

Department, Christie Hospital NHS Trust, Wilmslow Road, Manchester M20 4BX, UK

E Anderson

Department of Pathology, Western General Hospital, Edinburgh EH4 2XU, UK

$\mathrm{T}$ Anderson

Area Laboratories, Crosshouse Hospital, Kilmarnock KA2 0BE, UK

R Adamson

Department of Histopathology, UCL Medical School, Rockefeller Building, University Street, London WC1E 6JJ, UK A Rhodes

K Miller

Department of Pathology, Clinical Sciences Building, Leicester Royal

Infirmary, PO Box 65, Leicester LE2 7LX, UK $\mathrm{R}$ Walker

Correspondence to: Professor Leake e-mail:

R.Leake@bio.gla.ac.uk

Accepted for publication 20 January 2000

\begin{abstract}
The biochemical assay for the oestrogen receptor has shown the clinical value of knowing the concentration of the receptor within tissue. The immunohistochemical assay is rapidly taking over from the biochemical assay. Therefore, it is vital to have an equivalent scoring system that will have the same predictive value. This paper reports both a practical protocol and a scoring system that should achieve this aim. This approach should be applicable to many more biomarkers detected by immunohistochemistry.

(f Clin Pathol 2000;53:634-635)
\end{abstract}

Keywords: breast cancer; oestrogen receptor; tamoxifen; immunohistochemistry; external quality control

The overview of adjuvant breast cancer treatments ${ }^{1}$ confirmed the importance of measuring oestrogen receptor values in breast cancer biopsies. The results showed that benefit was proportional to the quantity of receptor present. The overview was based largely on oestrogen receptor values that had been determined by the biochemical assay. The switch to an immunohistochemical assay requires the establishment of a protocol that will allow at least semiquantitative reporting of results, which can be subject to external quality control. ${ }^{2}$

The protocol described in this paper reflects an overall method that is currently working well in several UK and mainland European laboratories. Using this methodology, these laboratories find that results (obtained with the attached scoring system) are highly reproducible. Such a scoring system also correlates well with the old biochemical assays and provides equally significant predictive and prognostic information. ${ }^{3}$

To keep loss of antigenicity to a minimum, excised tissue should be incised (sliced) and placed into an adequate volume of fixative (preferably, formal saline or neutral buffered formalin) for about 24 hours (slicing allows the necessary rapid and even penetration of fixative-the fixation time can vary according to the size of the tissue slices, but should be judged on the achievement of good morphological appearance). Wherever possible, adjacent sections of normal parenchyma should be taken to act as an internal positive control. Localisation biopsies should be handled ac- cording to the published guidelines. ${ }^{4}$ Core biopsies may be fixed for a shorter period than 24 hours.

Sections should be heated on to slides that are either commercially precoated or hand coated with a strong adhesive, such as APES, to avoid sections becoming detached from the slide during antigen retrieval. Antigen retrieval is most reliably achieved with one to two minutes in a pressure cooker using $0.01 \mathrm{M}$ citrate buffer, pH 6.0 (although properly controlled microwaving is acceptable and detailed methodology for antigen retrieval, buffer selection, etc is available from UK NEQAS-ICC (address below)). The antireceptor antibodies selected should be those that have been validated and have a good record in published work-for example, clone 1D5 (available from Dako (Ely, Cambridgeshire, UK) and others) or clone 6F11 from Novocastra (Vector Labs, Peterborough, UK) for the oestrogen receptor, and clones 1A6 (Novocastra) or PR88 (Biogenix, Menarini Diagnostics, Finch-Hampstead, Berkshire, UK) for the progesterone receptor. The use of surfactants in buffers before the application of the antireceptor antibody can dramatically reduce the detection of the receptor greatly (which has been shown-for example, for 1D5) and, therefore, should be avoided.

In house controls should come from a composite block containing receptor rich, receptor poor, and receptor negative components. If, as recommended, normal breast epithelial cells in adjacent tissue have been taken, these will provide a good internal, positive control.

Staining of sections by hand can be more reliable for low, positive receptor expression. Where automatic stainers are used, it is essential to check the equivalence of the sensitivity with that produced by manual methods. Experienced users of automatic stainers can obtain good results even for low positive receptor expression, as long as surfactants are avoided. A sensitive method of detection, such as the avidin-biotin-complex (for example, the Dako Duet K0492 gives consistently good results but others will work equally well) should be used. Counterstaining should not obscure weak, positive staining and, when this is avoided, can be valuable. Excessive counterstaining does occur in some automatic stainers although adjustment should be possible. True receptor staining is taken to be nuclear and cytoplasmic staining should be ignored.

All laboratories must take part in an external quality assessment (EQA) scheme and an 
Table 1 Suggested scoring system

\begin{tabular}{ll}
\hline Score for proportion staining & Score for staining intensity \\
\hline $0=$ No nuclear staining & $0=$ No staining \\
$1=<1 \%$ nuclei staining & $1=$ Weak staining \\
$2=1-10 \%$ nuclei staining & $2=$ Moderate staining \\
$3=11-33 \%$ nuclei staining & $3=$ Strong staining \\
$4=34-66 \%$ nuclei staining & \\
$5=67-100 \%$ nuclei staining &
\end{tabular}

Adding the two scores together gives a maximum score of 8 .

appropriate one is run by UK NEQAS (address below).

In reporting the results of steroid receptor immunohistochemistry, simple scoring systems are found to work best. ${ }^{2}$ These use either a direct count of the proportion of epithelial nuclei that take up stain or a simple combination of the proportion of cells staining plus a measure of intensity of stain - for example, the "quick score".

Table 1 shows a simple, additive scoring system that is gives a range from 0 to 8 , which has proved to be highly reproducible among readers.

Experience to date ${ }^{3}$ suggests that, using such a simple scoring system, appropriate cut off values for treatment of advanced disease are:

- A score of zero indicates that endocrine treatments will definitely not work.

- A score of 2 or 3 indicates a small $(20 \%)$ chance of response to endocrine treatments.

- A score of 4-6 indicates an even (50\%) chance of response.

- A score of 7 or 8 indicates a good (75\%) chance of response.

- Where progesterone receptor content has also been determined, endocrine treatment is deemed worthwhile in patients with low oestrogen receptor but high progesterone receptor values.

- In elderly patients, some tumour shrinkage may be seen after endocrine treatment, even though the staining for the oestrogen receptor is low.

- Perhaps the single, most valuable observation is that for patients with a breast cancer that is zero on the quick score, the chances of response to endocrine treatment are essentially nil and these patients should receive an alternative first line treatment.

- The appropriate cut off values for adjuvant treatment, using steroid receptor determination by immunohistochemistry have yet to be determined, although Harvey and colleagues $^{3}$ reported that, using a similar scoring system but slightly different protocol, a value $>2$ was the optimal cut off point for predicting improved outcome $(\mathrm{p}<0.0001)$.

Technical advice can be obtained from: UK NEQAS for Immunohistochemistry, Department of Histopathology, UCL Medical School, University Street, London WC1E 6JJ, tel: +440207 209 6049; fax: +44 0207387 3674; email: rmkdhcr@ucl.ac.uk.

1 Early Breast Cancer Trialists Collaborative Group. Tamoxifen for early breast cancer: an overview of Tamoxifen for early breast cancer: an
randomised trials. Lancet 1998;351: 1451-67.

2 Barnes DM, Millis RR, Beex LVAAM, et al. Increased use of immunohistochemistry for oestrogen receptor measurement in mammary carcinoma: the need for quality assurance. Eur F Cancer 1998;34:1677-82.

3 Harvey JM, Clark GM, Osborne CK, et al. Estrogen receptor status by immunohistochemistry is superior to the igand-binding assays for predicting response to adjuvant therapy in breast cancer. $\mathcal{F}$ Clin Oncol 1999;17:1474-85.

4 UK National Coordinating Group for Breast Screening Pathology. Pathology reporting in breast screening, 2 nd ed. NHSBSP Publication No. 3, April 1995. 